\title{
Different mechanisms in weight loss-induced blood pressure reduction between a calorie-restricted diet and exercise
}

\author{
Kazuko Masuo ${ }^{1,2}$, Hiromi Rakugi ${ }^{3}$, Toshio Ogihara ${ }^{3}$ and Gavin W Lambert ${ }^{1,4}$
}

The present study compared the effectiveness of a mild calorie-restricted diet (D) alone, exercise (EX) alone and a combination of D+EX on weight loss-induced blood pressure (BP) reduction over 24 weeks. We focussed especially on the relationship between sympathetic nervous activity, as indicated from measures of plasma norepinephrine (NE), and insulin resistance (homeostasis model of insulin resistance, HOMA-IR). The three groups each comprised 30 obese, hypertensive men. Body mass index (BMI), fat mass, waist-to-hip ratio, BP, plasma NE and HOMA-IR were measured every 2 weeks during the first 4 weeks and subsequently every 4 weeks for the next 20 weeks. All basal parameters were similar among the three groups. At 24 weeks, the combination group with D+EX comprised a significantly higher prevalence of normotensive subjects than the $D$ alone or EX alone group $(P<0.05)$. In the $\mathrm{D}$ alone group, plasma NE was decreased significantly at 2 weeks, reductions in BMI, fat mass and BP were observed at 8 weeks, and waist-to-hip ratios and HOMA-IR were decreased at 12 weeks. In comparison, in the EX alone group, significant reductions of fat mass and HOMA-IR were observed at 4 weeks. Plasma NE and HOMA-IR were reduced at 8 weeks while decreases in BP were detected at 12 weeks. In the D+EX group, significant reductions in plasma NE were observed at 2 weeks followed by significant decreases in BMI, fat mass, waist-to-hip ratio, BP levels and HOMA-IR at 4 weeks. The magnitudes of reductions of all parameters were greatest in the D+EX group. These results demonstrate that D+EX exerts a stronger ameliorative effect on weight loss, weight loss-induced BP reduction, normalization of BP, sympathetic activation and insulin resistance compared with D or EX alone. D and EX might, perhaps, exert different mechanisms on weight loss and weight loss-induced BP reduction; however, a combination of caloric restriction and exercise is preferred to control BP levels in obese hypertensive patients.

Hypertension Research (2012) 35, 41-47; doi:10.1038/hr.2011.134; published online 4 August 2011

Keywords: a calorie-restricted diet; an aerobic exercise; insulin resistance; obesity; sympathetic nervous activity

\section{INTRODUCTION}

Obesity has become a global epidemic over the past few decades largely because of changes in dietary habits, food composition and reduced physical activity. Obesity-related cardiovascular disease development has become a major cause of morbidity and mortality worldwide. With the westernization of the developing world this problem will escalate further. It is widely recognized that insulin resistance or hyperinsulinemia and sympathetic nervous activation relates to obesity and hypertension, ${ }^{1-5}$ and these are known to be independent risk factors for future cardiovascular events. ${ }^{6-9}$

Weight loss is an important preventative measure and is associated with a reduction in all-cause mortality. ${ }^{10}$ Weight loss associated with lifestyle modifications such as diet and regular exercise are important and remain the first-line treatment for obesity and obesity-related hypertension. ${ }^{11-15}$ Whelton et al. ${ }^{16}$ reported in a meta-analysis of 54 randomized, controlled trials comprising 2419 participants that aerobic exercise was associated with a significant reduction in mean systolic and diastolic blood pressure (BP) in both overweight and normal-weight participants regardless of whether they were hypertensive or normotensive on admission to the investigation. A number of studies, including a series of the Dietary Approaches to Stop Hypertension (DASH) diet studies, ${ }^{17-19}$ showed that not only the dietary pattern, but also aerobic exercise and calorie restriction were necessary for weight loss, weight loss-induced BP reduction and improvement of insulin sensitivity. Weight loss with life-style modifications can result in significantly better BP control among hypertensive patients with pharmacological treatments. ${ }^{15,20}$ Although both life-style modifications (that is, calorie-restricted diet and exercise) are recommended for weight loss, it has not been fully clarified which is more effective with regards to weight loss-induced BP reduction in obese hypertensive patients. Therefore, in the present study, we sought to clarify the differences in neurohormonal mechanisms in weight loss-induced

\footnotetext{
${ }^{1}$ Human Neurotransmitters Laboratory, Baker IDI Heart \& Diabetes Institute, Melbourne, Victoria, Australia; ${ }^{2}$ Nucleus Network Ltd, Baker IDI Heart \& Diabetes Institute, Melbourne, Victoria, Australia; ${ }^{3}$ Department of Geriatric Medicine and Nephrology, Osaka University Graduate School of Medicine, Suita, Osaka, Japan and ${ }^{4}$ Faculty of Medicine, Nursing and Health Sciences, Monash University, Melbourne, Victoria, Australia

Correspondence: Professor K Masuo, Human Neurotransmitters Laboratory, Baker IDI Heart \& Diabetes Institute, 75 Commercial Road, Melbourne, Victoria 3004 , Australia. E-mail: kazuko.masuo@bakeridi.edu.au

Received 18 February 2011; revised 20 May 2011; accepted 17 June 2011; published online 4 August 2011
} 
$\mathrm{BP}$ reduction between mild caloric restrictive diet and exercise over a 24-week period. In addition, we observed which lifestyle modification was the more effective in normalizing BP in obese hypertensive patients.

\section{METHODS}

\section{Subjects}

Subjects were recruited from a cohort of 1121 Japanese men who worked in a single company in Osaka, Japan. Recruitment occurred during one of their biannual medical evaluations. Their education and socio-economic status were very similar. Ninety overweight or obese (BMI $\left.>25 \mathrm{~kg} \mathrm{~m}^{-2}\right)$, grade I hypertensive $(140 / 90 \leqslant B P<160 / 100 \mathrm{~mm} \mathrm{Hg})$ Japanese men were enrolled in this study. The subjects were randomly divided into three groups, weight loss alone, exercise alone and weight loss and exercise. Subjects were free from other clinical conditions including diabetes (fasting blood glucose level $<100 \mathrm{mg} \mathrm{dl}^{-1}$ and $\mathrm{HBA}_{1 \mathrm{c}}<6.0 \%$ ), psychological or emotional problems, ${ }^{21}$ or obstructive sleep apnea. ${ }^{22}$ Although participants were hypertensive, no subjects were taking antihypertensive agents or any other medications. Only the subjects who had not changed their diet, exercise or body weight ( $>5 \%$ provided in their biannual medical evaluation records) over the previous 6 months, including the run-in period of 1 month, were eligible for enrollment. ${ }^{23,24}$ Randomization was stratified according to basal BP and body mass index (BMI). Average age at the baseline period was $38 \pm 5$ years for the diet alone group, $37 \pm 6$ years for exercise alone group and $38 \pm 5$ years for the combination of diet and exercise group. All subjects had an average caloric intake between 2200 and $2600 \mathrm{kcal}$, with $8-12 \mathrm{~g} \mathrm{NaCl}$ per day and no additional physical activity in the 4 weeks before enrollment (baseline), which were confirmed from their meal and exercise monitoring diaries. The protocol was approved by the Ethics Committee of Osaka University Graduate School of Medicine, Japan, and written informed consent was obtained from all the subjects.

\section{Study design}

The weight-loss programs comprised (i) a low-caloric (1760-1840 kcal (22-23 units) per day, $55 \%$ of calories from carbohydrate, $30 \%$ from protein and $15 \%$ from fat) and low-sodium diet ( $7 \mathrm{~g} \mathrm{NaCl}$ per day), (ii) aerobic exercise of $>1 \mathrm{~h}$ daily, for example, walking, jogging or gym exercise with original $(2400 \mathrm{kcal}$ per day) diet and (iii) combined mild calorie restricted diet as mentioned above and exercise. The subjects attended a 1-h private teaching and counseling session each week for four weeks, followed by biweekly $1 \mathrm{~h}$ sessions for 23 additional months. All sessions were led by experts in nutrition and exercise counseling. Calorie intake was calculated based on the subjects' meal diary and was controlled by a nutritionist. The physical activity was quantified and recorded by the use of step-counters, which were used on a daily basis. Diet and exercise compliance were monitored according to the subjects' own records every 2 weeks and were monitored at private counseling sessions. Compliance to diet and exercise was considered excellent and consistent based on those records. None of the participants withdrew from the study.

\section{Measurements}

Data including height, body weight, BMI, percentage total body fat mass, waist circumference, waist-to-hip ratio and urinalysis were determined in the morning after an overnight fast of $12 \mathrm{~h}$. After $30 \mathrm{~min}$ rest in the supine position, $\mathrm{BP}$, heart rate and venous blood sampling for measurements of blood glucose, plasma norepinephrine (NE), insulin and leptin were obtained. Samples were taken at baseline, every 2 weeks for the first 4 weeks, and every 4 weeks for the following 20 weeks. BP and heart rate were measured $>3$ times in the supine position by an automated sphygmomanometer (TM-2713, A\&D, Tokyo, Japan) using an appropriately sized cuff based on arm circumference. Recorded BP and heart rates were averaged. The percentage body fat mass was determined by impedance measurements (BF-102, Tanita, Tokyo, Japan). Total body fat mass $(\mathrm{kg})$ was calculated according to the following formula: (percentage body fat mass $(\%) / 100) \times$ body weight $(\mathrm{kg})$. The homeostasis model assessment of insulin resistance (HOMA-IR): fasting glucose $\left(\mathrm{mg} \mathrm{dl}^{-1}\right)$ $\times$ fasting insulin $\left(\mu \mathrm{Uml}^{-1}\right) / 405 .{ }^{25}$ Plasma $\mathrm{NE}$, insulin and leptin were measured as previously reported. ${ }^{23}$

\section{Statistical analyses}

Values are shown as mean \pm s.d. All data analyses were performed using SPSS 8.0 for Windows (Chicago, IL, USA). Prevalence of hypertension was estimated using the $\chi^{2}$ test. Differences in the same group were examined by the paired $t$-test and among groups were examined using one-way ANOVA with the Holms-Sidak method for post hoc analysis. Multiple regression linear analyses were applied to evaluate the relationship between changes in BP from basal to 24 weeks as a dependent variable and changes in BMI, total body fat mass, plasma NE, HOMA-IR and leptin as independent variables. In addition, to evaluate the contributions of neurohormonal responses during the acute phase (4 weeks) and weight loss-induced BP reduction at 24 weeks, multiple regression analyses were examined using changes in BMI and BP from baseline to 24 weeks as dependent variables and changes in plasma NE, HOMA-IR and leptin from baseline to 4 weeks as independent variables.

\section{RESULTS}

Diet (calorie and salt intake) and exercise (steps per day assessed with pedometers) compliance were assessed from the subjects' own records every 2 weeks, which were recorded at private counseling sessions. Compliance to diet and exercise during the 6-month period was considered excellent based on examination of diaries, which were monitored by experts in exercise and nutrition. Depending on the groups allocated, there occurred a stable calorie and salt intake, and physical activity (steps per day); that is, no significant differences in calorie intake and salt intake between the diet (D) alone group and the group in combination of diet and exercise (D+EX), and no significant differences in physical activity and salt intake between the exercise (EX) alone group and the D+EX group.

All subjects were pre-hypertensive or grade I hypertensive ( $\geqslant 140$ or $\geqslant 90 \mathrm{~mm} \mathrm{Hg}$ ) at the baseline period but at 24 weeks the group with the combination of D+EX included the highest prevalence of normotensive subjects ( $<140$ and $<90 \mathrm{~mm} \mathrm{Hg}$ ) (D+EX group, $n=24$; the $\mathrm{D}$ alone group, $n=14$; the EX alone group, $\left.n=9 ; \chi^{2}=13.92, P<0.05\right)$ (Table 1). BP reduction over 24 weeks was significantly greater in the $\mathrm{D}+\mathrm{EX}$ group compared with the $\mathrm{D}$ alone or EX alone groups $(P<0.05)$, and the D alone group had a slightly greater reduction in systolic BP than the EX alone group.

At the baseline period, all parameters including BMI, total body fat mass, waist-to-hip ratio, BP, plasma NE, insulin and leptin levels were similar among the three study groups (Table 1). At 24 weeks, BMI, total body fat mass and BP levels were significantly less in the combined $\mathrm{D}+\mathrm{EX}$ group than in the $\mathrm{D}$ alone or EX alone groups. Additionally, at 24 weeks, total body fat mass and waist-to-hip ratio were significantly less in the EX alone group compared with those in the D alone group, although BMI in the D alone group was slightly lower than the EX alone group (Table 1). Plasma NE and leptin levels in the group with combination D+EX at 24 weeks were less compared with those in the D alone or EX alone group, but HOMA-IR in the D+EX group and in the EX alone group was smaller than the $\mathrm{D}$ alone group. In the $\mathrm{D}$ alone group, plasma NE levels were smaller than the EX alone group throughout the weight-loss protocol, but HOMA-IR was greater in the D alone group than the EX alone group. Changes in plasma leptin were similar to changes in total body fat mass in all three groups (Table 2).

There were significant different patterns of changes in plasma NE and HOMA-IR between the D alone and EX alone groups even though absolute weight loss or percent weight loss, especially during the first 12 weeks, were similar. Significant weight loss was recognized at 8 weeks in both groups and in both groups BMI decreased gradually during the 8 -week period. In the $\mathrm{D}$ alone group, significant reduction in plasma NE was observed at 2 weeks before significant weight loss or BP reduction, and HOMA-IR reduction was only observed after significant weight loss. In the EX alone group, a significant reduction 
Table 1 Comparisons of characteristics between the diet alone, exercise alone and combination of diet and exercise groups

\begin{tabular}{|c|c|c|c|c|c|c|}
\hline & Baseline & 2 weeks & 4 weeks & 8 weeks & 12 weeks & 24 weeks \\
\hline Diet alone & $0 / 30$ & $0 / 30$ & $1 / 29$ & $4 / 26$ & $9 / 21^{* * *}$ & $14 / 16^{* * *}$ \\
\hline Diet+exercise & $0 / 30$ & $0 / 30$ & $6 / 24$ & $12 / 18$ & $19 / 11^{* * * * *}$ & $24 / 6^{* * * * *}$ \\
\hline \multicolumn{7}{|l|}{$B M I\left(\mathrm{~kg} \mathrm{~m}^{-2}\right)$} \\
\hline Diet+exercise & $30.5 \pm 1.8$ & $28.1 \pm 2.0$ & $26.8 \pm 2.1^{*}$ & $25.7 \pm 2.0^{* *}$ & $24.8 \pm 2.2^{* *}$ & $24.0 \pm 2.1^{* *}$ \\
\hline \multicolumn{7}{|l|}{ Body weight (kg) } \\
\hline Diet alone & $97.8 \pm 4.9$ & $93.6 \pm 6.2$ & $90.4 \pm 4.9$ & $86.8 \pm 6.2^{*}$ & $83.9 \pm 6.8^{* *}$ & $81.6 \pm 6.5^{* *}$ \\
\hline Exercise alone & $100.7 \pm 5.6$ & $96.7 \pm 6.0$ & $94.4 \pm 6.6$ & $90.8 \pm 6.0^{*}$ & $87.8 \pm 5.3^{* *}$ & $87.1 \pm 6.0^{* *}$ \\
\hline Diet+exercise & $98.8 \pm 5.8$ & $91.0 \pm 6.5$ & $86.8 \pm 6.8^{*}$ & $83.3 \pm 6.5^{* *}$ & $80.4 \pm 7.1^{* *}$ & $77.8 \pm 6.8$ \\
\hline \multicolumn{7}{|l|}{ Total fat mass ( $\mathrm{kg}$ ) } \\
\hline Diet+exercise & $30.8 \pm 6.1$ & $27.5 \pm 5.6$ & $22.3 \pm 5.7^{* *, * * * * *}$ & $17.8 \pm 5.8^{* *, * * * * *}$ & $16.3 \pm 5.3^{* *}$ & $15.1 \pm 5.4^{* *}$ \\
\hline \multicolumn{7}{|l|}{ Waist-to-hip ratio } \\
\hline Diet alone & $1.09 \pm 0.05$ & $1.05 \pm 0.05$ & $1.03 \pm 0.06$ & $0.99 \pm 0.08$ & $0.98 \pm 0.05^{*}$ & $0.95 \pm 0.05^{*, * * *, * * * * *}$ \\
\hline Exercise alone & $1.11 \pm 0.09$ & $1.05 \pm 0.06$ & $1.01 \pm 0.05$ & $0.98 \pm 0.05^{*}$ & $0.97 \pm 0.05^{*}$ & $0.91 \pm 0.05^{* *}$ \\
\hline Diet+exercise & $1.09 \pm 0.06$ & $1.04 \pm 0.05$ & $0.99 \pm 0.06^{*}$ & $0.94 \pm 0.05^{*}$ & $0.93 \pm 0.06^{*}$ & $0.91 \pm 0.06^{*}$ \\
\hline \multicolumn{7}{|c|}{ Systolic BP (mm Hg) } \\
\hline Diet alone & $154 \pm 4$ & $153 \pm 5$ & $153 \pm 4^{* * *}$ & $149 \pm 5^{*, * * *}$ & $146 \pm 6^{*}$ & $141 \pm 5^{* *}$ \\
\hline Exercise alone & $155 \pm 6$ & $154 \pm 6$ & $154 \pm 4^{* * *}$ & $152 \pm 5^{* * *}$ & $149 \pm 4^{*, * * * *}$ & $145 \pm 6^{* *, * * *}$ \\
\hline Diet+exercise & $156 \pm 6$ & $150 \pm 5^{*}$ & $145 \pm 6^{*}$ & $141 \pm 5^{* *}$ & $138 \pm 7^{* *}$ & $136 \pm 6^{* *}$ \\
\hline \multicolumn{7}{|c|}{ Diastolic BP $(\mathrm{mm} \mathrm{Hg})$} \\
\hline \multicolumn{7}{|l|}{ Mean $B P(m m ~ H g)$} \\
\hline Exercise alone & $117 \pm 6$ & $116 \pm 6$ & $114 \pm 5$ & $111 \pm 6$ & $109 \pm 5^{*, * * *}$ & $106 \pm 6^{* *, * * *}$ \\
\hline Diet+exercise & $117 \pm 5$ & $111 \pm 5$ & $106 \pm 7^{*}$ & $103 \pm 5^{* *}$ & $100 \pm 7^{* *}$ & $99 \pm 6^{* *}$ \\
\hline \multicolumn{7}{|l|}{ Heart rate (b.p.m.) } \\
\hline Diet alone & $78 \pm 5$ & $80 \pm 6$ & $75 \pm 6$ & $73 \pm 5$ & $72 \pm 4^{*}$ & $70 \pm 5^{*}$ \\
\hline Exercise alone & $80 \pm 5$ & $78 \pm 6$ & $75 \pm 7$ & $73 \pm 5^{*}$ & $69 \pm 5^{* *}$ & $66 \pm 6^{* *}$ \\
\hline Diet+exercise & $80 \pm 5$ & $74 \pm 6$ & $72 \pm 6^{*}$ & $69 \pm 6^{* *}$ & $67 \pm 5^{* *}$ & $65 \pm 7^{* *}$ \\
\hline
\end{tabular}

Abbreviations: BMI, body mass index; BP, blood pressure; $\mathrm{HT}$, hypertension ( $\geqslant 140$ or $\geqslant 90 \mathrm{~mm} \mathrm{Hg}$; $\mathrm{NT}$, normotension $(<140 \mathrm{and}<90 \mathrm{~mm} \mathrm{Hg}$.

Parentheses show percent values compared to basal values.

${ }^{*} P<0.05$.

$* * P<0.01$ vs. the values at the baseline period.

$* * * P<0.05$.

$* * * * P<0.01$ vs. the group with a combination of diet and exercise.

$* * * * * P<0.05$.

$* * * * * * P<0.01$ vs. the group with exercise alone.

in HOMA-IR was noted at 4 weeks before significant weight loss and reductions in plasma NE were observed; significant weight loss was evident at 8 weeks. Therefore, one could speculate that a calorie restricted diet might lead to a normalization or suppression of the sympathetic overactivity associated with the BP reduction accompanying weight loss and lead to an amelioration of insulin resistance. On the other hand, the exercise only program might lead to reduction in total fat mass with amelioration of HOMA-IR at 4 weeks and then significant reduction in plasma NE at 8 weeks. The significant BP reduction occurred subsequently at 12 weeks (Table 2 ).

The magnitude of reductions in BMI, total body fat mass, BP, heart rate, level of plasma NE or leptin, and HOMA-IR over 24 weeks were greater in the group with combined D+EX compared with the groups with D alone or EX alone. 
Table 2 Comparisons of neurohormonal parameters between the diet alone, exercise alone and combination of diet and exercise groups

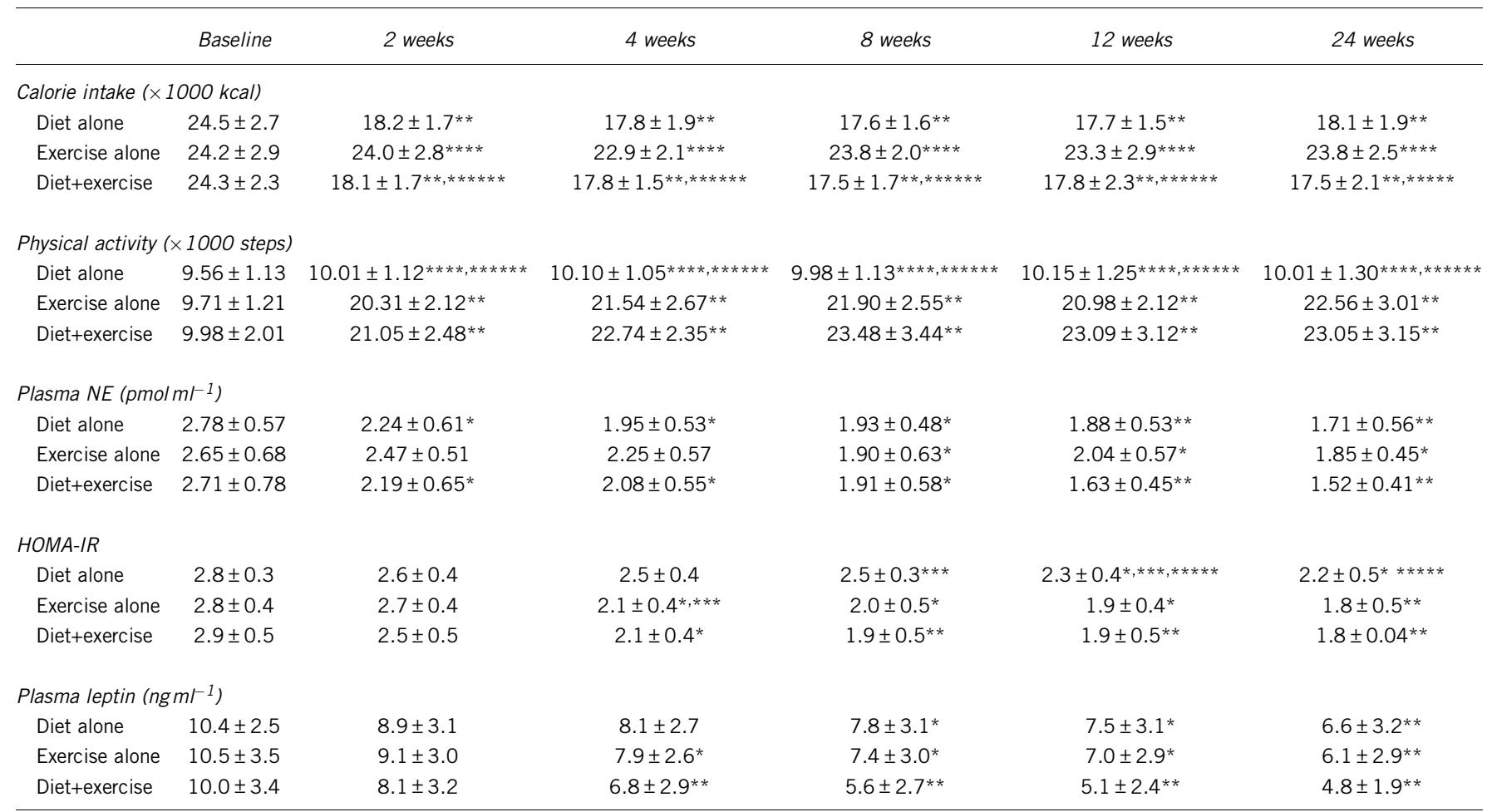

Abbreviations: HOMA-IR, homeostasis model of insulin resistance; NE, norepinephrine.

Parentheses show percent values compared to basal values.

${ }^{*} P<0.05$.

${ }^{*} P<0.01$ vs. the values at the baseline period.

$* * * P<0.05$.

$* * * * P<0.01$ vs. the group with a combination of diet and exercise.

$* * * * P<0.01$ vs
$* * * * * P<0.05$

$* * * * * P<0.01$ vs. the group with exercise alone.

Regarding the order of reductions in all parameters, in the D alone group, plasma NE decreased significantly at 2 weeks followed by significant reduction in body weight, BP and leptin at 8 weeks. HOMA-IR and waist-to-hip ratios decreased significantly at 12 weeks. On the other hand, in the EX alone group, significant decreases in HOMA-IR, leptin and total body fat mass were observed initially at 4 weeks (before significant plasma NE reductions), and plasma NE at 8 weeks. BP reduction was evident at 12 weeks. In the combined D+EX group, significant reduction in plasma NE was observed at 2 weeks, and significant reductions in HOMA-IR, leptin, BMI, total body fat mass, waist-to-hip ratio and BP occurred at 4 weeks, earlier than the D alone or EX alone groups (Table 2).

In multiple regression analyses, changes over 24 weeks in plasma $\mathrm{NE}$ ( $P=0.042$ for weight loss; $P=0.039$ for $\mathrm{BP}$ reduction), changes in HOMA-IR ( $P=0.043, P=0.067$, respectively) and changes in leptin $(P=0.038, P=0.054)$ were significant determinants of weight loss $\left(R^{2}=0.3221, \quad P=0.047\right)$ and changes in mean BP $\left(R^{2}=0.2821\right.$, $P=0.053)$. Changes in plasma NE over the first 4 weeks $(P=0.040$ for weight change; $P=0.032$ for $\mathrm{BP}$ reduction), changes in HOMA-IR over 4 weeks $(P=0.048, P=0.061)$ and changes in plasma leptin over 4 weeks $(P=0.046, P=0.054)$ were significant determinants for changes in BMI over 24 weeks $\left(R^{2}=0.3889, P=0.041\right)$ and BP reduction over 24 weeks $\left(R^{2}=0.3051, P=0.049\right)$.

\section{DISCUSSION}

Weight loss is associated with substantial health benefits. In this investigation, we highlight that the mode of weight loss influences different physiological pathways, with calorie restriction initially targeting the sympathetic nervous system, whereas changes in insulin resistance precede effects on the sympathetic nervous system in subjects partaking in an exercise program. Indeed, the novel findings of this study are that in the $\mathrm{D}$ alone group, suppression of high plasma NE followed by reductions in HOMA-IR, weight loss and BP reduction was observed. This contrasted with the EX alone group, where reduction in HOMA-IR was observed initially followed by suppression of high plasma NE, weight loss and BP reduction. Additionally, in all subjects, not only the $\mathrm{D}$ alone group, changes in plasma NE and HOMA-IR in the acute period of weight loss (4 weeks) were significant determinants of changes in BMI over the 24-week period, suggesting that early reductions on sympathetic nervous activity and insulin resistance might be predictors of subsequent weight loss. On the other hand, only reductions on sympathetic nervous activity were related with BP reduction during weight-loss program. Importantly, the observation that no one withdrew from this study indicates that a mild calorie restricted diet plus light exercise (mainly walking $>1 \mathrm{~h}$ per day) with frequent counseling ${ }^{24}$ may be a beneficial clinical approach in achieving and maintaining long-term weight control.

Other major findings were that: (i) as previously demonstrated, the combination therapy of D and EX is associated with a more prevalent normalization of hypertension in obese hypertensive subjects compared with D or EX alone and (ii) greater and earlier suppression of sympathetic overactivity and reduction in HOMA-IR were noted in the combination group than in the group with D or EX alone. Owing to the higher success rate of normalization of $\mathrm{BP}$, these findings 
support the recommendation of the combination protocol for weight loss with mild caloric restriction and exercise rather than one of them for obesity hypertension.

The problem of obesity is now recognized as a global epidemic. ${ }^{26,27}$ Importantly, hypertension and its associated co-morbidities are frequently associated with obese status. ${ }^{28}$ Abdominal obesity and weight gain are documented as independent cardiovascular risk factors, due in part to their association with insulin resistance and sympathetic nervous activation. ${ }^{29,30}$ Weight-loss studies with lifestyle modifications involving a low-calorie diet and exercise $e^{31-33}$ as well as bariatric surgery ${ }^{34,35}$ provide some evidence that intentional weight loss has long-term benefits on all cause mortality in overweight/obese adults. Therefore, weight loss is recommended as the first-line treatment for obesity. Giving some insight into the possible physiological pathways linking increased morbidity and mortality, elevated sympathetic nervous activity, ${ }^{36-38}$ insulin resistance ${ }^{31,32}$ and stimulation of the renin-angiotensin-aldosterone system ${ }^{39}$ are ameliorated after weight loss.

The sympathetic nervous system has an important role in the regulation of energy expenditure, and reduced energy expenditure and resting metabolic rate are predictive of weight gain. Acute fat load intravenously ${ }^{39}$ and oral glucose ingestion ${ }^{40,41}$ significantly increased $\mathrm{BP}$ and activated the sympathetic nervous system in obese, but otherwise healthy, subjects. Considerable evidence derived from experiments documenting reduction in plasma $\mathrm{NE},{ }^{42-46}$ urinary NE, whole-body NE spillover ${ }^{47}$ and muscle sympathetic nerve activity indicates that calorie restricted diet-induced weight loss is sympatho-inhibitory. ${ }^{48}$ Similarly, exercise interventions alone have been shown to lower whole-body NE spillover by $24 \%$ and renal NE spillover by $41 \%$ in healthy young men independent of changes in body weight. ${ }^{49}$ Sodium intake is also known to influence sympathetic nervous activity ${ }^{50}$ and BP levels, ${ }^{51}$ however, in the present study, all groups were matched in sodium intake and were maintained on a mild sodium restricted diet.

A number of investigations ${ }^{17-19,52,53}$ have demonstrated the importance of exercise on weight loss and weight loss-induced BP reduction, however, few studies have examined the longitudinal differences in the mechanisms of weight loss between D alone and D+EX. Our recent study in obese Caucasian individuals with the metabolic syndrome demonstrated that exercise over 12 weeks had additional effects on weight loss and BP reduction and were associated with suppression of plasma renin activity compared with calorie restriction alone, whereas suppression of muscle sympathetic nervous activity was similar. ${ }^{54}$ In another study, comparing the effects of exercise and a calorie restricted diet on sympathetic nervous activity, the addition of moderateintensity aerobic exercise training to a weight-loss program did not confer additional benefits on resting sympathetic nervous activity as assessed from measures of whole-body NE spillover and muscle sympathetic nerve activity, although BP, baroreflex sensitivity and metabolic parameters improved significantly but similarly between the two study groups. ${ }^{52}$ These studies demonstrated the importance of insulin resistance and the renin-angiotensin system for weight lossinduced BP reduction associated with aerobic exercise, however, these studies did not compare neurohormonal changes during the acute phase of weight loss. In the present study, at the early stage of weight loss, before significant weight loss at 8 weeks, suppression on plasma NE followed by a decrease in HOMA-IR and BP reduction was observed in the $\mathrm{D}$ alone group, whereas significant reduction in HOMA-IR followed by suppression on sympathetic nervous activity and $\mathrm{BP}$ reduction occurred in the EX alone group. Weight loss was similar between the D alone and EX alone groups. These findings indicate that the neurohormonal response to weight-loss programs differs between a calorie restricted diet and exercise. Sowers et al. ${ }^{43}$ followed plasma epinephrine, NE and dopamine during an 8-week period of a low-caloric protein diet. Plasma NE and epinephrine declined during the first 2 weeks of caloric restriction before significant weight loss. Previously, we examined responses of plasma NE, HOMA-IR, body weight and BP levels to a very low-caloric diet ( $1000 \mathrm{kcal}$ per day) over 14 days. ${ }^{45}$

Although weight loss before 4 weeks was similar among the three study groups, the neurohormonal changes and BP reduction pattern were different. It should be noted that the diminution in plasma NE was observed before weight loss or BP reduction in the three study groups, and hence may be attributed to the effects of negative energy balance in all groups, suggesting that suppression of sympathetic activation by weight-loss programs, either caloric restriction or exercise, may have an important role in weight loss-induced BP reduction. In our previous study in obese Caucasian subjects with the metabolic syndrome, weight loss over 12 weeks with D+EX was associated with significantly greater decreases in total body fat mass, abdominal fat mass and waist-to-hip ratios than the D alone group, but reduction in plasma NE and NE spillover were greater in the D alone group compared with the combination group with $\mathrm{D}+\mathrm{EX}^{52}$ The groups, including those in the EX program, had earlier and stronger improvements on HOMA-IR. ${ }^{52}$ Taken together, a calorie restricted diet, but not aerobic exercise, is a prime mover in sympathetic nervous adaptation, and aerobic exercise may have a major role in the improvement of insulin resistance. A calorie restricted diet and exercise may exert different mechanisms on weight loss, weight lossinduced BP reduction and insulin resistance.

Interestingly, some investigators ${ }^{55}$ reported that obese subjects without the metabolic syndrome had more pronounced benefits of weight loss-induced BP reduction than those with the metabolic syndrome. We previously observed blunted responses of plasma NE and insulin to oral glucose ingestion in subjects with insulin resistance, ${ }^{40,56}$ suggesting that exercise amelioration on insulin resistance may be an ancillary effect on weight loss-induced BP reduction. In addition, several investigators ${ }^{57-59}$ compared the effects of BP and forearm vascular conductance measured using venous occlusion plethysmography between D and D+EX groups. Both groups experienced similar effects on weight loss and $\mathrm{BP}$ reductions, although the forearm vascular conductance responses (improved muscle vasodilation) during exercise and mental stress increased significantly and was normalized only in the D+EX group, thereby demonstrating that EX exerts additional effects and improves vascular dilation. These investigations highlight the importance of aerobic exercise in weight-loss programs in order to significantly reduce BP and improve insulin resistance.

\section{CONCLUSIONS}

Obesity is an independent risk factor for cardiovascular disease development. Weight loss is the first treatment in obesity and obesity-related hypertension, however, few studies have investigated the differences in mechanisms of $\mathrm{D}$ alone, EX alone and a combination of $\mathrm{D}+\mathrm{EX}$ on weight loss-induced $\mathrm{BP}$ reduction. In the present study, we compared total body fat mass, waist-to-hip ratio, BP, plasma NE (as an index of sympathetic nervous activity), HOMA-IR (as an index of insulin resistance) and plasma leptin levels among three weight-loss protocols: low-caloric diet alone, exercise alone and a combination of low-caloric diet and exercise over 24 weeks in obese, hypertensive Japanese men. The weight-loss protocol with a combination of D+EX had the strongest ameliorative effect on weight loss, 
being especially effective on abdominal obesity, BP reduction, normalization of BP, sympathetic nervous activation and insulin resistance. A low-caloric diet and exercise operated via different mechanisms on weight loss-induced BP reduction; however, both protocols exerted favorable effects on sympathetic activation and insulin resistance.

In conclusion, a combination therapy for weight loss with a lowcaloric diet and exercise is recommended for weight-loss protocols owing to the higher prevalence of normalization of $\mathrm{BP}$ and stronger suppression of sympathetic activation and insulin resistance, both of which are known as risk factors for cardiovascular events. Different mechanisms may contribute to BP reduction associated with weight loss with the relevant physiological mechanism at play being dependent on the mode of weight loss. Suppression of sympathetic activation appears to be the prime mover in initiating the ameliorative effect on insulin resistance and BP reduction during a calorie restricted diet, whereas aerobic exercise initially influences insulin resistance independently of suppression of sympathetic activation. For clinical implications, a mild calorie-restricted diet plus light exercise, comprising walking $>1 \mathrm{~h}$ per day for instance, with frequent counseling ${ }^{24}$ may be beneficial as a clinical approach in achieving and maintaining longterm weight control and resultant BP normalization.

\section{CONFLICT OF INTEREST}

The authors declare no conflict of interest.

\section{ACKNOWLEDGEMENTS}

Dr Lambert is supported by a National Health and Medical Research Council of Australia Research Fellowship. The laboratory of Dr Lambert currently receives research funding from private organizations including ARDIAN Inc, Allergan, Abbott (formerly Solvay) Pharmaceuticals and Scientific Intake. These organizations had no role in this manuscript. The investigator reports no conflicts of interest with regards to this work.

1 Grassi G, Seravalle G, Cattaneo BM, Bolla GB, Lanfranchi A, Colombo M, Glannattasio C, Brunani A, Cavagnini F, Mancia G. Sympathetic activation in obese normotensive subjects. Hypertension 1995; 25(Pt 1): 560-563.

2 Grassi G. Role of the sympathetic nervous system in human hypertension. $J$ Hypertens 1998; 16(Pt 2): 1979-1987.

3 Landsberg L. Insulin-mediated sympathetic stimulation: role in the pathogenesis of obesity-related hypertension (or, how insulin affects blood pressure, and why). $J$ Hypertens 2001; 15: 523-528.

4 Masuo K, Katsuya T, Fu Y, Rakugi H, Ogihara T, Tuck ML. 2- and $\beta 3$-adrenergic receptor polymorphisms are related to the onset of weight gain and blood pressure elevation over 5 years. Circulation 2005; 111: 3429-3434.

5 Masuo K. Role of beta2- and beta3-adrenoceptor polymorphisms in hypertension and metabolic syndrome. Int J Hypertens 2010; 2010: 832821.

6 Esler M, Kaye D. Sympathetic nervous system activation in essential hypertension, cardiac failure and psychosomatic heart disease. J Cardiovasc Pharmacol 2000; 35(Suppl 4): S1-S7.

7 Masuo K, Katsuya T, Sugimoto K, Kawaguchi H, Rakugi H, Ogihara T, Tuck ML. High plasma norepinephrine levels associated with beta2-adrenoceptor polymorphisms predict future renal damage in nonobese normotensive individuals. Hypertens Res 2007; 30: 503-511.

8 Ksiazek A, Zatuska W. Sympathetic overactivity in uremia. J Ren Nutr 2008; 18 : $118-121$.

9 Zoccali C, Mallamaci F, Parlongo S, Cutrupi S, Benedetto FA, Tripepi G, Bonanno G, Rapisarda F, Fatuzzo P, Seminara G, Cataliotti A, Stancanelli B, Malatino LS. Plasma norepinephrine predicts survival and incident cardiovascular events in patients with end-stage renal disease. Circulation 2002; 105: 1354-1359.

10 Berglund G, Nilsson P, Eriksson KF, Nilsson JA, Hedblad B, Kristenson H, Lindgrade F. Long-term outcome of the Malmo preventive project: mortality and cardiovascular morbidity. J Intern Med 2000; 247: 19-29.

11 National Heart, Lung and Blood Institute, National Institute of Health. The Seventh Report of the Joint National Committee on "Prevention, Detection, Evaluation, and Treatment of High Blood Pressure (JNC 7). http://www.nhlbi.nih.gov/guidelines/hypertension/\#status.

$12 \mathrm{JSH}$ 2009. JSH guideline for the management of hypertension. Hypertens Res 2009; 32: 11-23.

13 Hackam DG, Khan NA, Hemmelgrarn BR, Rabkin SW, Touyz RM, Campbell NR, Padwal R, Campbell TS, Lindsay MP, Hill MD, Quinn RR, Mahon JL, Herman RJ, Schiffrin EL,
Ruzicka M, Larochelle P, Feldman RD, Lebel M, Poirier L, Arnold JM, Moe GW, Howlett JG, Trudeau L, Bacon SL, Petrella RJ, Milot A, Stone JA, Drouin D, Boulanger JM, Sharma M, Hamet P, Fodor G, Dresser GK, Carruthers SG, Pylypchuk G, Burgess ED, Burns KD, Vallée M, Prasad GV, Gilbert RE, Leiter LA, Jones C, Ogilvie RI, Woo V, McFarlane PA, Hegele RA, Tobe SW;. The 2010 Canadian Hypertension Education Program recommendations for the management of hypertension: part 2-therapy. Can $\mathrm{J}$ Cardiol 2010; 26: 249-258.

14 Goodpaster BH, Delany JP, Otto AD, Kuller L, Vockley J, South-Paul JE, Thomas SB, Brown J, McTigue K, Hames KC, Lang W, Jakicic JM. Effects of diet and physical activity interventions on weight loss and cardiometabolic risk factors in severely obese adults: a randomized trial. JAMA 2010; 304: 1795-1802.

15 Miller III ER, Erlinger TP, Young DR, Jehn M, Charleston J, Rhodes D, Wasan SK, Appel LJ. Results of the diet, exercise, and weight loss intervention trial (DEW-IT). Hypertension 2002; 40: 612-618.

16 Whelton SP, Chin A, Xin X, He J. Effect of aerobic exercise on blood pressure: a metaanalysis of randomized, controlled trials. An Intern Med 2002; 136: 493-503.

17 Blumenthal JA, Babyak MA, Sherwood A, Craighead L, Lin PH, Johnson J, Watkins LL, Wang JT, Kuhn C, Feinglos M, Hinderliter A. Effects of dietary approaches to stop hypertension diet alone and in combination with exercise and caloric restriction on insulin sensitivity and lipids. Hypertension 2010; 55: 1199-1205.

18 Smith PJ, Blumenthal JA, Babyak MA, Craighead L, Welsh-Bohmer KA, Browndyke JN, Strauman TA, Sherwood A. Effects of the dietary approaches to stop hypertension diet, exercise, and caloric restriction on neurocognition in overweight adults with high blood pressure. Hypertension 2010; 55: 1331-1336.

19 Himderliter AL, Babyak MA, Sherwood A, Blumenthal JA. The DASH diet and insulin sensitivity. Curr Hypertens Rep 2011; 13: 67-73.

20 Masuo K, Mikami H, Ogihara T, Tuck ML. Weight reduction and pharmacological treatment in obese hypertensives. Am J Hypertens 2001; 14(Pt 1): 530-538.

21 Claesson IM, Josefsson A, Sydsjo G. Prevalence of anxiety and depressive symptoms among obese pregnant and postpartum women: an intervention study. BMC Public Health 2010; 10: 766.

22 Adesseun GA, Rosas SE. The impact of obstructive sleep apnea on chronic kidney disease. Curr Hypertens Rep 2010; 12: 378-383.

23 Masuo K, Mikami H, Ogihara T, Tuck ML. Weight gain-induced blood pressure elevation. Hypertension 2000; 35: 1135-1140.

24 Lambert GW, Masuo K, Dixon JB. Weight loss in obesity and metabolic syndrome. Curr Hypertens Rev 2011; 6: 130-138.

25 Yokoyama H, Emoto M, Fujiwara S, Motoyama K, Morioka T, Komatsu M, Tahara H, Shoji T, Okuno Y, Nishizawa Y. Quantitative insulin sensitivity check index and the reciprocal index of homeostasis model assessment in normal range weight and moderately obese type 2 diabetic patients. Diabetes Care 2003; 26: 2426-2432.

26 Mokdad AH, Serdula MK, Dietz WH, Bowman BA, Marks JS, Koplan JP. The spread of the obesity epidemic in the United States, 1991-1998. JAMA 1999; 282: 1519-1522.

27 Lee DS, Chiu M, Manuel DG, Tu K, Wang X, Austin PC, Mattern MY, Mitiku TF, Svenson LW, Putnam QW, Flanagan WM, Tu JV. Trends in risk factors for cardiovascular disease in Canada: temporal, socio-demographic and geographic factors. CMAJ 2009; 191: E55-E66.

28 Mokdad AH, Ford ES, Bowman BA, Diez WH, Vinicor F, Bales VS, Marks JS. Prevalence of obesity, diabetes, and obesity-related health risk factors, 2001. JAMA 2003; 289: 76-79.

29 Mancia G, Bombelli M, Facchetti R, Madotto F, Corrao G, Trevano FS, Giannattasio C, Grassi G, Sega R. Long-term risk of diabetes, hypertension and left ventricular hypertrophy associated with the metabolic syndrome in a general population. J Hypertens 2008; 26: 1602-1611.

30 Cameron AJ, Dustan DW, Owen N, Zimmet PZ, Barr EL, Tonkin AM, Magliano DJ, Murray SG, Welborn TA, Shaw JE. Health and mortality consequences of abdominal obesity: evidence from the AusDiab study. Med J Aust 2009; 191: 202-208.

31 Orchard TJ, Temprosa M, Goldberg R, Haffner S, Ratner R, Marcovina S, Fowler S. The effect of metformin and intensive lifestyle intervention on the metabolic syndrome: the Diabetes Prevention Program randomized trial. Am Intern Med 2005; 142: 611-619.

32 Anderssen SA, Carroll S, Urdal P, Holme I. Combined diet and exercise intervention reverses the metabolic syndrome in middle-aged males: results from the Oslo Diet and Exercise Study. Scand J Med Sci Sports 2007; 17: 687-695.

33 Poobalan AS, Aucott LS, Smith WCS, Avenell A, Jung R, Broom J. Long-term weight loss effects on all cause mortality in overweight/obese populations. Obes Rev 2007; 8: 503-513.

34 Adams TD, Gress RE, Smith SC, Halverson RC, Simper SC, Rosamond WD, Lamonte MJ, Stroup AM, Hunt SC. Long-term mortality after gastric bypass surgery. N Engl J Med 2007; 357: 753-761.

35 Dixon JB, O'Brien PE, Playfair J, Chapman L, Schachter LM, Skinner S, Proietto J, Bailey M, Anderson M. Adjustable gastric banding and conventional therapy for type 2 diabetes: a randomized controlled trial. JAMA 2008; 299: 316-323.

36 Lambert E, Straznicky N, Schlaich MP, Esler M, Dawood T, Hotchkin E, Lambert G. Different pattern of sympathoexcitation in normal-weight and obesity-related hypertension. Hypertension 2007; 50: 862-868.

37 Straznicky NE, Grima MT, Lambert EA, Eikelis N, Dawood T, Lambert GW, Nestle PJ, Masuo K, Sari Cl, Chopra R, Mariani JA, Schlaich MP. Exercise augments weight loss induced improvement in renal function in obese metabolic syndrome individuals. J Hypertens 2011; 29: 553-564.

38 Esler M, Jennings G, Lambert G. Noradrenaline release and the patho-physiology of primary human hypertension. Am J Hypertens 1989; 2: S140-S146. 
39 Gosmanov AR, Smiley DD, Robalino G, Siquiera J, Khan B, Le NA, Patel RS, Quyyumi AA, Peng L, Kitabchi AE, Umpierrez GE. Effects of oral and intravenous fat load on blood pressure, endothelial function, sympathetic activity, and oxidative stress in obese healthy subjects. Am J Physiol Endocrinol Metab 2010; 299: E953-E958.

40 Masuo K, Katsuya T, Ogihara T, Tuck ML. Acute hyperinsulinemia reduces plasma leptin levels in insulin-sensitive Japanese men. Am J Hypertens 2005; 18(2 Pt 1): 235-243.

41 Straznicky N, Lambert GW, Masuo K, Dawood T, Eikelis N, Nestel PJ, McGrane MT, Mariani JA, Socratous F, Chopra R, Esler MD, Schlaich MP, Lambert EA. Blunted sympathetic neural response to oral glucose in obesity subjects with the insulinresistant metabolic syndrome. Am J Clin Nutr 2009; 89: 27-36.

42 Masuo K, Mikami H, Ogihara T, Tuck ML. Differences in mechanisms between weight loss-sensitive and -resistant blood pressure reduction in obese subjects. Hypertens Res 2001; 24: 371-376.

43 Sowers JR, Nyby M, Stern N, Beck F, Baron S, Catania R, Vlachis N. Blood pressure and hormone changes associated with weight reduction in the obese. Hypertension 1982 4: 686-691.

44 Maxwell MH, Heber D, Waks AU, Tuck ML. Role of insulin and norepinephrine in the hypertension of obesity. Am J Hypertens 1994; 7: 402-408.

45 Masuo K, Ogihara T. Mechanisms in obesity-related hypertension in humans. Hypertension 2000; 36(Suppl 2): 17 (Proceeding for Jackson Cardiovascular Renal Meeting).

46 Gohler L, Hahnemann T, Michael N, Oehme P, Steglich HD, Conradi E, Grune T, Siems WG. Reduction of plasma catecholamines in humans during clinically controlled severe underfeeding. Prev Med 2000; 30: 95-102.

47 Straznicky NE, Lambert EA, Lambert GW, Masuo K, Esler MD, Nestle PJ. Effects of dietary weight loss on sympathetic activity and cardiac risk factors associated with the metabolic syndrome. J Clin Endocrinol Metab 2005; 90: 5998-6005.

48 Sivitz WI, Fink BD, Morgan DA, Fox JM, Donohouse PA, Haynes WG. Sympathetic inhibition, leptin, and uncoupling protein subtype expression in normal fasting rats. Am J Physiol 1999; 277(Pt 1): E668-E677.

49 Meredith IT, Friberg P, Jennings GL, Dewar EM, Fazio VA, Lambert GW, Esler MD. Exercise training lowers resting renal but not cardiac sympathetic activity in humans. Hypertension 1991; 18: 575-582.

50 Masuo K, Ogihara T, Kumahara Y, Yamatodani A, Wada H. Plasma norepinephrine and dietary sodium intake in normal subjects and patients with essential hypertension. Hypertension 1983; 5: 767-771.
51 Maxwell MH, Kushiro T, Dornfeld LP, Tuck ML, Waks AU. BP changes in obese hypertensive subjects during rapid weight loss. Comparison of restricted $\mathrm{v}$ unchanged salt intake. Arch Intern Med 1984; 144: 1581-1584.

52 Straznicky NE, Lambert EA, Nestel PJ, McGrane MT, Dawood T, Schlaich MP, Masuo K, Eikelis N, de Courten B, Mariani JA, Esler MD, Socratous F, Chopra R, Sari Cl, Paul E, Lambert GW. Sympathetic neural adaptation in hypocaloric diet with or without exercise training in obese metabolic syndrome subjects. Diabetes 2010; 50: 71-79.

53 Blumenthal JA, Babyak MA, Hinderliter A, Watkins LL, Craighead L, Lin PH, Caccia C, Johnson J, Waugh R, Sherwood A. Effects of the DASH diet alone and in combination with exercise and weight loss on blood pressure and cardiovascular biomarkers in men and women with high blood pressure: the ENCORE study. Arch Intern Med 2010; 170: 126-135.

54 Strazanicky NE, Grima MT, Lambert EA, Eikelis N, Dawood T, Lambert GW, Nestel PJ, Masuo K, Sari Cl, Chopra R, Mariani JA, Schlaich MP. Exercise augments weight loss induced improvement in renal function in obese metabolic syndrome individuals. J Hypertens 2011; 29: 553-564.

55 Pescatello LS, Blanchard BE, Van Heest JL, Maresh CM, Gordish-Dressman H, Thompson PD. The metabolic syndrome and the immediate antihypertensive effects of aerobic exercise: a randomised control design. BMC Cardiovascular Disorders 2008; 8: 12 doi:10.1186/1471-2261-8-12.

56 Straznicky NE, Lambert GW, McGrane MT, Masuo K, Dawood T, Nestel PJ, Eikelis N, Schlaich MP, Esler MD, Socratous F, Chopra R, Lambert EA. Weight loss may reverse blunted sympathetic neural responsiveness to glucose ingestion in obese subjects with metabolic syndrome. Diabetes 2009; 58: 1126-1132.

57 Ribeiro MM, Silva AG, Santos NS, Guazzelle I, Matos LN, Trombetta IC, Halpern A, Negrao CE, Villares SM. Diet and exercise training restore blood pressure and vasodilatory responses during physiological maneuvers in obese children. Circulation 2005; 111: 1915-1923.

58 Trombetta IC, Batalha LT, Rondon MU, Laterza MC, Kuniyoshi FH, Gowdak MM, Barretto AC, Halpern A, Villares SM, Negrao CE. Weight loss improves neurovascular and muscle metaboreflex control in obesity. Am J Physiol Heart Circ Physiol 2003; 11: H974-H982.

59 Tonacio AC, Trombetta IC, Rondon MU, Batalha LT, Kuniyoshi FH, Laterza MC, Suzuki PH, Gowdak MM, Barretto AC, Halpern A, Villares SM, Negrao CE. Effects of diet and exercise training on neurovascular control during mental stress in obese women. Braz J Med Biol Res 2006; 39: 53-62. 